\title{
Factors Associated with the Recovery of Walking Ability Following Total Hip Arthroplasty
}

\author{
MANABU NANKAKU ${ }^{1)}$, TADAO TSUBOYAMA ${ }^{2)}$, HARUHIKO AKIYAMA ${ }^{3)}$, JUN NiSHIMURA ${ }^{1)}$, \\ YASUKO FUJITA $^{1)}$, HARUNA KAWAI ${ }^{1)}$, TAKASHI NAKAMURA ${ }^{3)}$, RYOSUKE KAKINOKI ${ }^{1)}$ \\ 1) Rehabilitation Unit, Kyoto University Hospital: 54 Kawahara-cho, Shogoin, Sakyo-ku, Kyoto 606- \\ 8507, Japan.TEL: +8175-751-3571,E-mail:nankaku@kuhp.kyoto-u.ac.jp \\ 2) Human Health Sciences, Graduate School of Medicine, Kyoto University \\ 3) Department of Orthropedic Surgery, Faculty of Medicine, Kyoto University
}

\begin{abstract}
Purpose] The aim of this study was to identify the factors related to the recovery of walking ability at the early stage after total hip arthroplasty (THA). [Subjects and Methods] The subjects of this study were 99 patients who had undergone unilateral THA. The hip abductor and knee extensor strengths were measured and the timed up and go (TUG) test was performed prior to THA. The number of days to achieve gait with a cane, an index of earlyphase improvement in walking after THA, was retrospectively examined. [Results] A stepwise multiple regression analysis was performed with the number of days to achieve gait with a cane as the dependent variable, and other measurements as independent variables. The preoperative physical functions correlated with the number of days to achieve gait with a cane following THA. The stepwise multiple regression analysis selected three factors (preoperative TUG test, surgical approach, and mobility at the beginning of transfer training) as the significant variables affecting the number of days to achieve gait with a cane following THA. [Conclusion] The results of the present study suggest that preoperative TUG, surgical approach and mobility at the start of transfer training are useful indicators for predicting ambulation ability in the early stage after THA.
\end{abstract}

Key words: Total hip arthroplasty, Prognosis, Walking ability

(This article was submitted Apr. 1, 2011, and was accepted Apr. 26, 2011)

\section{INTRODUCTION}

Many clinics have recently introduced clinical paths for patients recovering from total hip arthroplasty (THA). The goal of introducing such paths is to standardize quality in medical care and to reduce the length of hospital stay postTHA. Indeed, clinical paths are useful treatment tools in the early postoperative phase of THA.

Physical therapy in the acute post-THA stage is now considered a standard treatment. The aim of early physiotherapy is to maximize patients' functionality and independence in performing activities of daily living (ADL) and to prevent complications such as deep vein thrombosis, pulmonary embolism, muscle atrophy, and hip dislocation ${ }^{1)}$. Prediction of postoperative ambulatory ability is critical for determining the length of hospital stay, and can also aid patients in developing appropriate expectations in terms of regaining the ability to perform ADL in the early postoperative stage of THA.

Some studies have reported that the physical function before surgery and surgical technique are robust predictors of walking ability after THA and total knee arthroplasty ${ }^{2-6)}$. However, few studies have sought to identify the factors associated with the recovery of walking ability during the acute postoperative period following THA.

The purpose of this study was to identify the factors predicting the number of days to achieve cane-assisted gait following THA surgery.

\section{SUBJECTS AND METHODS}

A total of 99 patients ( 84 women and 15 men) with a mean age of $60.3 \pm 11.1$ years (range: $35-81$ ) and a mean body mass index (BMI) of $22.6 \pm 3.7 \mathrm{~kg} / \mathrm{m}^{2}$ (range: $15.7-$ 33.7) participated in this study. All patients underwent primary THA due to unilateral osteoarthritis of the hip at the department of orthopedic surgery of Kyoto University Hospital, between April 2008 and March 2010. Exclusion criteria included the occurrence of postoperative complications, a leg length discrepancy of more than $3 \mathrm{~cm}$, bilateral hip osteoarthritis, revision THA, and rheumatoid arthritis.

THA surgery was performed utilizing Dall's direct lateral approach (65 cases) or the modified mini-one anterolateral incision approach (MMIS) (34 cases). Weight bearing transfer training began on the third postoperative day and all patients received a prescribed 4 week rehabilitation program in the hospital. All patients were able to walk independently with a cane at the time of discharge.

This study was approved by the Ethics Committee of Kyoto University Graduate School and Faculty of Medicine. All patients were informed about the study 
procedures before testing and provided their written informed consent.

Hip abductor and knee extensor strength on the affected side were measured prior to surgery. Hip abductor strength was measured with a hand-held dynamometer (Nihon Medix Co., Ltd., Matsudo, Japan) during a 3 second isometric contraction with manual resistance. Patients rested while supine with both the hip and knee in the neutral position. A force sensor was placed $5 \mathrm{~cm}$ above the lateral epicondyle of the femur. Knee extensor strength was measured using the IsoForce GT-330 (OG Giken Co., Ltd., Okayama, Japan) during a 3 second isometric contraction. With the patient in a sitting position with the hip and knee at $90^{\circ}$ angles, a force sensor was placed $5 \mathrm{~cm}$ above the ankle joint. Torque was calculated by multiplying strength by the lever arm and expressed as a percentage of body weight $(\mathrm{Nm} / \mathrm{kg})$.

The timed up and go (TUG) test ${ }^{7)}$ was conducted to assess ambulatory ability. The TUG test measures the time (in seconds) it takes a subjects to rise from an armless chair (chair seat height $=45 \mathrm{~cm}$ ), walk for $3 \mathrm{~m}$ to a line drawn on the floor, and return to sitting position on the chair. The test was performed with the patients wearing shoes and walking at maximum speed without any assistive device.

During the initial post-operative transfer training, we classified patients into those who required physical assistance by another person (dependent transfer) or those who could transfer from the bed to wheelchair, and vice versa, with the use of an assistive device (independent transfer). The number of days to achieve gait with a cane, an index of early-phase improvement in walking after surgery, was retrospectively retrieved from the medical record of each patient. The number of days to achieve gait with a cane was defined as the number of days after surgery each patient needed to obtain ambulatory ability with T-cane support to move from the bed to the toilet of the hospital ward.

The best value of two trials of pre-operative physical function was used in the analysis. The level of association between the number of days to achieve gait with a cane and preoperative physical functions was first examined with Pearson correlation coefficients. Subsequently, multiple regression analysis was performed with the number of days to achieve gait with a cane as a dependent variable, and preoperative physical functions, age, gender, BMI, surgical
Table 1. Pearson's coefficient of correlation for the number of days to achieve gait with a cane after THA and preoperative physical functions

\begin{tabular}{lc}
\hline Preoperative physical functions & Correlation coefficients \\
\hline Hip abductor strength $(\mathrm{Nm} / \mathrm{kg})^{*}$ & -0.33 \\
Knee extensor strength $(\mathrm{Nm} / \mathrm{kg})^{*}$ & -0.38 \\
Timed up and go test $(\mathrm{sec})^{*}$ & 0.53 \\
\hline
\end{tabular}

$* \mathrm{p}<0.01$.

approach and dependent/independent mobility at the beginning of transfer training as independent variables. Statistical analysis was performed using SPSS for Windows, with $p<0.05$, considered statistically significant.

\section{RESULTS}

The mean preoperative hip abductor and knee extensor strengths on the affected side were $0.49 \pm 0.21$ (range: $0.12-1.35$ ) $\mathrm{Nm} / \mathrm{kg}$ and $1.44 \pm 0.56$ (range: $0.39-3.56) \mathrm{Nm} /$ $\mathrm{kg}$, respectively. The mean result of the TUG test prior to THA was $9.98 \pm 4.73(4.26-37.30)$ seconds. The average number of days to achieve gait with a cane after THA was $12.5 \pm 5.0$ (4-28) day. At the initial transfer training (bed to/from wheelchair) post-surgery, 50 patients were categorized as independent while the remaining 49 patients were categorized as dependent. Table 1 shows the Pearson correlation coefficients between the number of days to achieve gait with a cane after THA and preoperative physical function. The number of days to achieve gait with a cane following THA was positively correlated with preoperative hip abductor and knee extensor strengths on the affected side and the TUG test result. The multiple regression analysis identified preoperative TUG, surgical approach, dependent/independent mobility at the beginning of transfer training as key factors determining the number of days to achieve gait with a cane following THA (Table 2 ). Together, these factors explained $54.8 \%$ of the variability in the number of days to achieve gait with a cane following THA.

\section{DISCUSSION}

In general, the early postoperative physical therapy protocol consists of transfer training, muscle strengthening

Table 2. Results of the multiple regression analysis (factors determining the number of days to achieve gait with a cane following THA)

\begin{tabular}{lcc}
\hline Variables & Regression coefficient & Standard regression coefficient \\
\hline Age & 0.04 & 0.09 \\
Gender & 0.84 & 0.06 \\
BMI & 0.13 & 0.09 \\
Surgical approach* & -2.16 & -0.21 \\
Mobility at beginning of transfer training* & 4.12 & 0.42 \\
Hip abductor strength & -0.68 & -0.03 \\
Knee extensor strength & -0.97 & -0.11 \\
Timed up and go test* & 0.36 & 0.34 \\
\hline
\end{tabular}

${ }^{*} \mathrm{p}<0.01$. Multiple correlation coefficient adjusted for the degrees of freedom: $\mathrm{R}^{2}=0.55$. 
exercises, and gait training with some forms of external support on flat surfaces and stairs ${ }^{1)}$. The results of this study suggest that approximately $50 \%$ of THA patients can ambulate without requiring the assistance of another person during the initial transfer training session post-surgery. This study also found that the average number of days to achieve gait with a cane following THA was 12.5 days. This information may be valuable for planning rehabilitation programs during the acute postoperative period after THA surgery.

In the present study, the number of days to achieve gait with a cane following THA was positively correlated with preoperative physical function. These results indicate that superior preoperative physical status predicts fewer days will be necessary to walk with a cane following THA Specifically, stepwise multiple regression analysis suggested that the TUG test is most useful as a preoperative assessment tool for predicting the ambulation ability at an early stage after THA. It was reported that preoperative physical therapy, conducted for patients who have undergone THA, improves muscle strength and gait, allowing an early return to ambulatory function ${ }^{2,3)}$. Therefore, the preoperative practice of exercise programs, especially those including improvement of walking ability, may be important if patients are to regain the ability to perform ADL in the early stage after THA.

Recently, it has been reported that minimally invasive surgery approaches have shown superior results to those of conventional THA in terms of length of hospitalization, pain management and gait ability in the early postoperative period $^{4,5)}$. Our previous study showed that postoperative recovery of knee extensor strength was better with MMIS than the Dall approach, because the vastus lateralis muscle remains untouched in the former ${ }^{8)}$. Thus, the surgical approach is a factor that determines the number of days to achieve gait with a cane following THA.

Transfer training soon after surgery is recognized as an important element of any rehabilitation protocol for THA. Physical therapists teach the patients how to transfer from a wheelchair to a bed, thereby leading to a patient's early independence. The results of the present study suggest that mobility at the beginning of transfer training after surgery was related to the recovery of walking ability in the early stage after THA.

Our previous study showed that the number of days to achieve gait with a cane was related to the recovery of muscle strength at 4 weeks after THA $^{9)}$. Soon after THA surgery, patients begin to walk short distances in hospital rooms. The activity just after THA may promote the restoration of muscle function and walking ability. On the other hand, a higher level of physical activity may imply a greater risk of falls. Further research is needed to develop the screening methods designed to effectively identify patients at high risk of falling in the early stage after THA.

All patients in this study had unilateral osteoarthritis of the hip. The number of days to achieve gait with a cane for patients with bilateral osteoarthritis of the hip or revision THA surgery can be expected to be longer than that for those with unilateral osteoarthritis. It is desirable to perform a more detailed analysis of patients with bilateral hip osteoarthritis and revision THA surgery.

\section{REFERENCES}

1) Forrest GP, Roque JM, Dawodu ST: Decreasing length of stay after tota joint arthroplasty. Arch Phys Med Rehabil, 1999, 80: 192-194.

2) Foucher KC, Wimmer MA, Moisio KC, et al.: Time course and extent of functional recovery during the first postoperative year after minimally invasive total hip arthroplasty with two different surgical approaches-a randomized controlled trial. J Biomech, 2011, 44: 372-378.

3) Inaba Y, Dorr LD, Wan Z, et al.: Operative and patient care techniques for posterior mini-incision total hip arthroplasty. Clin Orthop Relat Res, 2005, 441: 104-114.

4) Rooks DS, Huang J, Bierbaum BE, et al.: Effect of preoperative exercise on measures of functional status in men and women undergoing total hip and knee arthroplasty. Arthritis Rheum, 2006, 55: 700-708.

5) Fortin PR, Clarke AE, Joseph L, et al.: Outcomes of total hip and knee replacement: preoperative functional status predicts outcomes at six months after surgery. Arthritis Rheum, 1999, 42: 1722-1728.

6) Kennedy DM, Hanna SE, Stratford PW, et al.: Preoperative function and gender predict pattern of functional recovery after hip and knee arthroplasty. J Arthroplasty, 2006, 21: 559-566.

7) Podsiadlo D, Richardson S: The timed 'Up \& Go': A test of basic functional mobility for frail elderly persons. J Am Geriatr Soc, 1991, 39: 142-148.

8) Aki K, Nankaku M, Nishimura J, et al.: Effect of difference in approach method on postoperative functional recovery after total hip arthroplasty. Hip Joint, 2010, 36: 134-136. (In Japanese)

9) Takagi A, Nankaku M, Akiyama $\mathrm{H}$, et al.: Effect of independence walking with assistive device on the recovery of muscle strength after total hip arthroplasty. Hip Joint, 2009, 35: 66-68 (In Japanese). 